this flow equals the patient's alveolar ventilation then all fresh gas participates in alveolar gas exchange, none is wasted, and fractional utilization is unity ( 100 per cent). In the current study, when rebreathing was detected, Humphrey et al, ${ }^{1}$ calculated the A.D.E. utilized 73 per cent of the fresh gas $\left(51.4 \pm 5.2 \mathrm{ml} \cdot \mathrm{kg}^{-1} \cdot \mathrm{min}^{-1}\right.$ FGF) compared to 51 per cent for the Magill circuit $\left(71.2 \pm 6.0 \mathrm{ml} \cdot \mathrm{kg}^{-1}\right.$. $\min ^{-1}$ FGF).

By adding tubing for an expiratory limb and moving the expiratory valve downstream the functional characteristics and efficiency of Mapleson' $\mathrm{s}^{3}$ original A system appear to have been improved. However do these lower flows and improved fractional utilization mean increased risks or benefits? A mean fresh gas flow value of $51.4 \pm 5.2$ $\mathrm{ml} \cdot \mathrm{kg}^{-1} \cdot \mathrm{min}^{-1}$ (mean $\pm \mathrm{SD}$ ) derived from ten patients does not guarantee that $\mathrm{CO}_{2}$ free inspired gas nor normal minute ventilation will be obtained when a standardized flow of $50 \mathrm{ml} \cdot \mathrm{kg}^{-1} \cdot \mathrm{min}^{-1}$ is used in every patient. Even though better utilization of fresh gas is achieved in this circuit, this standard deviation implies that some of these healthy patients would have been rebreathing $\mathrm{CO}_{2}$ if a FGF of only $50 \mathrm{ml} \cdot \mathrm{kg}^{-1} \cdot \mathrm{min}^{-1}$ had been used. One must recognize that the data imply that a FGF of $50 \mathrm{ml} \cdot \mathrm{kg}^{-1}$. $\mathrm{min}^{-1}$ prevents rebreathing. This is not an absolute guarantee, but rather an average approximation. The rule still must be "user beware" when selecting a FGF rate for spontaneous breathing.

What are the potential risks of using a convertible "universal" anaesthetic breathing system? Problems such as errors in lever placement could be avoided with experience. However, how is the "A mode" coaxial system checked for leaks? Since the reservoir bag is on the inspiratory side the Pethick test used for modified " $D$ " systems will not apply. Manual ventilation can only be achieved in the " $A$ mode," yet the exact fresh gas flow requirements, the amount of ventilation and other limitations have not been fully defined.

Humphrey's ingenuity and progress in simplifying the more complex dual lever system should be applauded. He has eliminated several sources of error and reduced the complexity of the system. As with other systems only in time will all problems be defined. In Canada there are no minimum safe standards of either function or structure of anaesthetic breathing systems. Canadian Standards Association (C.S.A.) approval is not necessary prior to marketing new breathing systems. C.S.A. guidelines for breathing systems are to be released in the near future. The Canadian Anaesthetists' Society and its members must address this issue to ensure elimination of faults before new equipment is used in Canada as we do for new pharmaceutical products. To state that technology should be made as safe as possible helps very little. The safety of machines can almost always be improved but, at some point, the price of safety is increasing complexity and cost.

A major message stressed by Humphrey et al. ${ }^{1}$ is that anaesthetists should choose their breathing system and FGF as carefully as they choose their anaesthetic drugs. The Humphrey A.D.E. system will be useful only if the clinical anaesthetist understands the functional characteristics of both the $\mathrm{A}$ and $\mathrm{D}$ mode and the limitations are fully documented.

\section{Aurions-nous un système de circuit anesthésique universel?}

Dans ce numéro du Journal, Humphrey et collègues décrivent une version du circuit anesthésique A.D.E. ${ }^{1,2}$ qui serait selon eux optimale pour tous les patients. Humphrey t $t$ al. ont démontré que le système "A.D.E. universel à bas flow" fonctionne comme un système D modifié (classification de Mapleson ${ }^{3}$ ) ou comme un circuit de Bain pour la ventilation contrôlée et lors de la mise en marche d'un interrupteur unique comme un système $A$ de Mapleson (ou circuit Lack) pour la respiration spontanée.

Les données présentes sont en accord avec les études préalables utilisant le mode $D$. En ventilation contrôlée (partie II) ${ }^{2}$ et mode D (A.D.E.) la $\mathrm{PCO}_{2}$ artérielle de dix patients était prévisible avec un flot de gaz frais de $70 \mathrm{ml} \cdot \mathrm{kg}^{-1} \cdot \mathrm{min}^{-1}$ et un volume courant de $10 \mathrm{ml} \cdot \mathrm{kg}^{-1}$ et une fréquence respiratoire de 12 à 15/minute (i.e., la ventilation contrôlée). 
Lors de la respiration spontanée (partie $)^{1}$ les auteurs suggèrent que la réinspiration peut être éliminée avec un flot de gaz frais de 50 $\mathrm{ml} \cdot \mathrm{kg}^{-1} \cdot \mathrm{min}^{-1}$ lorsque le système A.D.E. était utilisé en mode A (A.D.E.).

Plusieurs variables determinent la réinspiration en respiration spontanée. Ces considérations sont importantes pour un clinicien car comme $\mathrm{Nunn}^{4}$ l'énonce "il peut devenir presque impossible de déterminer la composition des gaz inspirés". Ainsi les cliniciens doivent se demander deux questions: 1) Est-ce que le système A.D.E. permet toujours un estimé prévisible des gaz inspirés assurant ainsi une anesthésie plus sécuritaire avec un flot de gaz frais économique de $50 \mathrm{ml} \cdot \mathrm{kg}^{-1} \cdot \mathrm{min}^{-1}$. 2) Quels sont les risques possibles qui peuvent altérer cet avantage?

Humphrey et al. ont étudie uniquement des patients en bonne santé (ASA classe I) respirant de l'halothane par un tube endotrachéal. L'influence d'autres facteurs pouvant affecter la réinspiration et par conséquent la tension du $\mathrm{CO}_{2}$ alvéolaire n'était pas évaluée. Ces facteurs comme l'anesthésie au masque qui augmentait l'espace mort, les changements de l'ondée respiratoire qui peuvent être altérés avec les différents agents anesthésiques, ${ }^{6}$ et l'effet de l'altération des résistances et des valves de sécurité à travers les circuits anesthésiques n'ont pas été considérés. L'application universelle d'un système de circuiterie unique requiert que cette technique soit également sécuritaire pour tous les patients lorsqu'on l'emploie avec un flot de gaz frais standardisé.

Quel est le seul sécuritaire de $\mathrm{CO}_{2}$ trouvé dans l'air inspiré et quel serait la meilleure technique de mesure pour déterminer la $\mathrm{PCO}_{2}$ inspirée? La conclusion de Humphrey et al. est basée sur la mesure d'echantillons à mi-flot de la bouche. Certains auteurs ${ }^{6}$ ont choisi de mesurer la $\mathrm{PCO}_{2}$ inspirée échantillonnée dans la carène afin de minimiser l'erreur d'échantillonnage et d'évaluer le volume du $\mathrm{CO}_{2}$ inspiré au delà des voies aériennes supérieures. Humphrey ${ }^{7}$ lui-même reconnaît que "la détermination du point auquel la réinspiration devient cliniquement significative continuera à présenter des problèmes." Il a choisi le point auquel la $\mathrm{PCO}_{2}$ inspiré atteinte au minimum $2 \mathrm{mmHg}(0.3$ pour cent). Conway s'est objecté à cette définition de la réinspiration. ${ }^{8}$ La définition de Humphrey signifie qu'une certaine quantité de $\mathrm{CO}_{2}$ est inspirée dans les voies aériennes supérieures tout au long de la phase inspiratoire et peut ainsi participer à l'échange gazeux. La quantité de $\mathrm{CO}_{2}$ qui peut atteindre les alvéoles n'a pas été déterminée dans cette étude. Est-ce que le degré de réinspiration est toujours associé avec une élévation de la $\mathrm{PaCO}_{2}$ sans altération d'autres facteurs? Avant qu'on puisse généraliser, qu'aucune réinspiration ne survient avec le circuit $A$.D.E. en mode $A$ avec un fot de $50 \mathrm{ml} \cdot \mathrm{kg}^{-1} \cdot \mathrm{min}^{-1}$ d'autres études sont nécessaires.

L'utilisation la plus efficace du flot de gaz frais dans n'importe quel circuit en respiration spontanée survient quand le flot est fixé de telle sorte qu'aucune réinspiration n'est détectée. Si ce flot est égale à la ventilation alvéolaire du patient, alors tout ce flot participe à l'échange gazeux alvéolaire et aucune partie n'est perdue le pourcentage d'utilisation étant de 100 pour cent. Dans la présente étude, quand la réinspiration est détectée, Humphrey et al. ' ont calculé que l'utilisation de gaz frais dans le système A.D.E. était de 73 pour cent $(51.4 \pm$ $5 \mathrm{ml} \cdot \mathrm{kg}^{-1} \cdot \mathrm{min}^{-1}$ ) comparativement à 51 pour cent dans le circuit Magill $\left(71.2 \pm 6.0 \mathrm{ml} \cdot \mathrm{kg}^{-1} \cdot \mathrm{min}^{-1}\right)$.

En ajoutant des tubes au côtés expiratoire et déplaçant la valve expiratoire en aval, les caractéristiques fonctionnelles et l'efficacité du circuit Mapleson original ${ }^{3}$ semblent être améliorés. Cependant est-ce que ces bas flots et de telles améliorations de la fraction utilisée signifient une augmentation du risque ou du benéfice? Le flot de gaz frais moyen de $51.4 \pm 5.2 \mathrm{ml} \cdot \mathrm{kg}^{-1} \cdot \mathrm{min}^{-1}$ (moyenne \pm SD) déterminé avec dix patients ne garantit pas l'absence de $\mathrm{CO}_{2}$ dans les gaz inspirés ou une ventilation minute normale quand un flot standard de $50 \mathrm{ml} \cdot \mathrm{kg}^{-1} \cdot \mathrm{min}^{-1}$ est utilisé chez tous les patients. Même si une meilleure utilisation des gas frais est obtenue dans le circuit, cette déviation standard implique que quelques patients sains ont pu réinspirer du $\mathrm{CO}_{2}$ si un flot de gaz frais de $50 \mathrm{ml} \cdot \mathrm{kg}^{-1} \cdot \mathrm{min}^{-1}$ a été utilisé. On doit reconnaitre que l'énoncé un flot de gaz frais de $50 \mathrm{ml}$. $\mathrm{kg}^{-1} \cdot \mathrm{min}^{-1}$ prévient la réinspiration. Ceci n'est pas une garantie absolue mais plutôt une moyenne approximative. La règle doit être "consommateur soyez prudent" quand vous choisissez un flot de gaz frais en respiration spontanée.

Quels sont les risques possibles lors de l'utilisation d'un système de circuit anesthésique universel convertible? Lers problèmes tels que les erreurs de positionnement du levier peuvent être évités avec l'expérience. Cependant comment peut-on vérifier 
les fuites dans le système A coaxial? Etant donné que le réservoir est sur le côté inspiratoire le test de Pethick utilisé pour le système $D$ modifié ne pourra $s$ 'appliquer. La ventilation manuelle peut seulement être accomplie en mode A mais ni la quantité de gaz frais ni la quantité de ventilation et autres facteurs limitants n'ont été bien définis.

L'ingéniosité et le progrès démontrés par Humphrey et al. dans la simplification du système à double levier doivent être reconnus. Il a éliminé plusieurs sources d'erreurs et réduit la complexité du système. Comme d'autres systèmes, c'est uniquement avec le temps que tous les problèmes seront définis. Au Canada il y n'y a aucune règle standard de sécurité minimale ni dans la fonction ni dans la structure des circuits anesthésiques. L'approbation de l'Association des Normes Canadiennes n'est pas nécessaire avant la mise en marché de nouveaux circuits anesthésiques. Les lignes directrices de la Société Canadienne des Anesthésistes concernant les circuits anesthésiques seront publiées dans le futur prochain. La Société Canadienne des Anesthésistes ainsi que ses membres doit s'attaquer à ce problème afin d'assurer l'élimination d'erreurs avant l'utilisation au Canada d'un nouvel équipement, de la même façon qu'on étudie les nouveaux produits pharmaceutiques. Le fait de dire que la technologie doit être accompagnée des normes les plus sécuritaires possibles n'avance à rien au problème. La sécurité des machines peut presque toujours être améliorée mais au-delà d'un certain point le prix de la sécurité est accompagné par une augmentation de la complexité et du coût du matériel.

Un message majeur important d'Humphrey est adressé aux anesthésistes pour qu'ils choisissent leurs circuits et leur flot de gaz frais avec autant de précaution qu'ils choisissent leurs drogues anesthésiques. Le système A.D.E. de Humphrey sera utile uniquement si l'anesthésiste clinique comprend les caractéristiques fonctionnelles des modes $\mathrm{A}$ et $\mathrm{D}$ et documentera leurs limitations.

\section{References}

1 Humphrey D, Brock-Utne JG, Downing JW. Single lever Humphrey A.D.E. low flow universal anaesthesia breathing system. Part I. Comparison with dual lever A.D.E., Magill and Bain systems in anaesthetized spontaneously breathing adults. Can Anaesth Soc J 1986; 33:6: 698-709.

2 Humphrey D, Brock-Utne JG, Downing JW. The Single lever Humphrey A.D.E. low flow universal anaesthesia breathing system. Part II. Comparison with Bain system in anaesthetized adults during controlled ventilation. Can Anaesth Soc J 1986; 33:6: 710-8.

3 Mapleson WW. The elimination of rebreathing in various semi-closed anaesthetic systems. $\mathrm{Br} \mathrm{J}$ Anaesth 1954; 26: 323-32.

4 Nunn JF. Applied Respiratory Physiology. Second Edition. Butterworths; London 1977. p. 233.

5 Kain ML, Panday $J, N u n n J F$. The effect of intubation on the dead space during halothane anaesthesia. Br J Anaesth 1969; 41: 94-101.

6 Byrick RJ, Janssen EG. Respiratory waveform and rebreathing in T-piece circuits: a comparison of enflurane and halothane waveforms. Anesthesiology 1980; 53: 371-8.

7 Humphrey D. Rebreathing and the Bain circuit. Anesthesiology 1983; 60: 80-1.

8 Conway CM. Anaesthetic Breathing Systems. Br J Anaesth 1985; 57: 649-57. 